\title{
Tratamiento de la onicofagia en niños. Revisión sistemática
}

\author{
Treatment of onycophagia in children: Systematic review
}

Claudia Cecilia Restrepo Serna

\section{Resumen}

Objetivo: Realizar una revisión sistemática para evaluar y analizar la evidencia científica disponible en terapias para la onicofagia en niños. Materiales y métodos: La literature se analizó a partir de Medline, Pubmed, ovid, EBSCO, ISI, Cochrane Library, Lylacs, Scielo, Scirus and the Internet, published from March 1985 to 2008. Se analizaron estudios de intervención o comparativos, ensayo clínico, metanálisis o estudio multicéntrico que incluyeran todas las terapias disponibles para resolver la Onicofagia en niños y niñas. Los niños y niñas incluidos en los estudios, debían presentar ausencia de otro tipo de hábitos y/o alteraciones neurológicas. Los estudios incluídos requerían haber hecho un diagnóstico inicial de Onicofagia a través de anamnesis y de ayudas diagnósticas. Resultados: De los 92 estudios revisados, uno cumplió los criterios de calidad. Los estudios seleccionados soportan las técnicas psicológicas para el tratamiento de la Onicofagia, más no el uso de aparatos intraorales o farmacoterapia en niños y niñas. Conclusión: Muy pocos estudios acerca de la terapia para la Onicofagia cumplen los criterios de calidad requeridos para la práctica basada en la evidencia. Aunque el estudio seleccionado cumplió con los criterios de calidad, no es suficiente para afirmar que existe adecuada evidencia para el tratamiento de la Onicofagia en niños y niñas, por lo que se requieren futuros estudios que comparen los diferentes tipos de terapia.

Palabras clave: Niños, Onicofagia, tratamiento, aparatos intraorales, terapia psicológica.

\section{Abstract}

Purpose: To conduct a systematic review to assess and analyze the scientific evidence about the available therapies for finger and nail biting in children. Materials and Methods: The literature was searched using Medline, Pubmed, ovid, EBSCO, ISI, Cochrane Library, Lylacs, Scielo, Scirus and the Internet, published from March 1985 to 2008. The inclusion criteria were: Investigations that treated finger or nail biting with any resource.. The investigations were required to be clinical trials or well done comparative studies. Metanalysis or multicenter studies were considered as well. The children included in the studies required not to have had other type of oral habits and / or neurological diseases and the diagnosis of or nail biting should be performed with anamnesis and other diagnostic tools. Results: From 92 records found, one fulfilled the inclusion criteria. It supports the psychological techniques to treat nail biting in children and do not support intraoral devices or pharmacology with the same objective. Conclusion: Although 
the selected study accomplished the quality criteria, it is not enough to affirm that treatment of nail biting in children is well supported. Treatment for nailbiting of onycophagia requires further studies.

Key words: children, onychophagia, treatment, psychological therapy, intraoral devices.

\section{Introducción}

La onicofagia (del griego onyx, 'uña' y phagein, 'comer') es el hábito de morderse (roer) o comerse las propias uñas ${ }^{1}$. Se presenta en estados de ansiedad y puede estar asociado con episodios de estrés ${ }^{1} \mathrm{o}$ alteraciones psiquiátricas ${ }^{2}$. Una vez establecido, las conductas que lo exacerban son hambre, aburrimiento e inactividad. Puede además estar asociado a trastornos mentales o emocionales, según su frecuencia ${ }^{2}$.

Aunque en muchos casos perdura hasta la edad adulta, la mayor incidencia de este mal hábito se da en la pubertad (44\%). Es más común en el sexo masculino que en el femenino. En niños de 7 a 10 años, ocurre entre el 28 y el $33 \%$ de los casos ${ }^{3}$.

Morderse las uñas puede resultar en infecciones cruzadas por el transporte de gérmenes que se ocultan bajo las uñas a la boca o viceversa ${ }^{4}$. La etiología es la diferencia en la flora bacteriana de ambas partes ${ }^{5}$.

El mordedor compulsivo de uñas puede estar tentado a comer no sólo ésta sino también la cutícula y la piel de alrededor, provocándose heridas por donde sufrir infecciones oportunistas micróbicas y virales ${ }^{6}$.

Dañarse la piel o uñas puede llevar a la ansiedad por la apariencia de las manos, produciéndose así un círculo vicioso (realimentación o feedback) que perpetúa la conducta.
El hábito crónico de morderse las uñas es perjudicial a largo plazo para la sustancia adamantina frontal de los dientes, aumentando la caries en las zonas afectadas ${ }^{5}$.

Se han planteado tratamientos psicológicos, farmacológicos y con mecanoterapia. La reacción de competencia por ejemplo, ha sido un tratamiento bastante usado en adultos con buena evidencia y resultados ${ }^{7}$ Sin embargo y a pesar de los daños que puede causar la onicofagia en la $\mathrm{ATM}^{8}$, dedosiv, dientes ${ }^{9}{ }^{10}$, etc., esta entidad no ha recibido la suficiente atención para basar el tratamiento clínico en la evidencia disponible.

Por lo anterior, el objetivo de esta revisión sistemática fue evaluar la evidencia disponible para el tratamiento de la onicofagia en niños y niñas.

\section{Material y métodos}

Criterios para considerar los estudios en la revisión:

\section{Tipo de estudios}

Se evaluaron estudios de intervención para onicofagia escritos en inglés, portugués o español. Fueron también considerados metanálisis o estudios multicéntricos.

\section{Tipo de participantes}

Niños y niñas de tres a diez años de edad, que presentaran onicofagia. Los sujetos debían presentar morfología facial normal, ausencia de síndromes o cualquier otra incapacidad neural o motora. Se descartaron aquellos estudios que consideraron enfermedades psiquiátricas asociadas al hábito. Mínimo, se requirió que a los sujetos se les hubiera realizado un cuestionario, 
una historia clínica completa e inspección intra y extraoral.

\section{Tipos de intervención}

Se consideró cualquier tipo de intervención para reducir o eliminar la onicofagia. Fueron evaluadas terapias farmacológicas, físicas y psicológicas. También se evaluó cualquier tipo de terapia alternativa o con aparatos intraorales.

\section{Tipos de medidas de resultados}

Se consideraron aquellos estudios que presentaran instrumentos validados y/o estandarizados como modelos de estudio, radiografías o cuestionarios.

El tipo de medida dependió exactamente de la intervención. Las medidas para el análisis fueron consideradas en dos dimensiones:

1. Medidas que indicaran la reducción del hábito.

2. Medidas que indicaran la eliminación completa de la onicofagia.

\section{Fuentes de información y estrategias de búsqueda}

Los estudios fueron identificados de las siguientes fuentes: Medline, Pubmed, Biomed Central, Ovid, Embase, EBSCO, ISI, Cochrane Library, Lylacs, Scielo, internet y las bases de datos del CES, Universidad de Antioquia, Universidad El Bosque, Universidad de Michigan y Universidad de Baylor. La literatura se revisó de Marzo de 1985 a 2008.

Se usaron los siguientes términos para identificar los artículos. Los términos MESH, se usaron de acuerdo a cada base de datos:
1. Singl*.

2. Doubl*.

3. Tripl $^{*}$.

4. Randomi*.

5. Clin*.

6. Trial $^{*}$.

7. $\left(\right.$ Clin $^{*}$ adj trial $\left.{ }^{*}\right)$.

8. (1 or 2 or 3$)$ and (5 and 6$)$.

9. Crossover.

10. Random*.

11. Allocate*.

12. RANDOM ASSIGNMENT.

13. Exp CLINICAL TRIALS.

14. Exp META ANALYSIS.

15. “CHILD [Mesh]".

16. Child ${ }^{*}$ or infant ${ }^{*}$ or boy* or girl* or preschool* $^{*}$ or schoolchild*.

17. “Child"[Mesh].

18. “Onychophagia"[MULTI].

19. "Nail Biting/drug therapy"[Mesh].

20. “Therapeutics"[Mesh].

21. “Physical Therapy Modalities"[Mesh].

22. "Cognitive Therapy"[Mesh].

23. "Psychoanalytic Therapy"[Mesh]).

24. "Homeopathy"[Mesh]. 
25. "Relaxation Techniques"[Mesh] "Psychotherapy, Group"[Mesh].

26. "Socioenvironmental Therapy"[Mesh] "Exercise Therapy"[Mesh].

27. "Myofunctional Therapy"[Mesh].

28. "NailBiting/prevention and control"[Mesh].

29. “Nail Biting/psychology"[Mesh].

30. “Nail Biting/rehabilitation"[Mesh].

31. "Nail Biting/therapy"[Mesh].

En internet se exploró con los siguientes motores de búsqueda: Metacrawler, Academic Google Academic, dogpile, Looksmart y Journal Sede.

Para Internet y las bases de datos del CES, Universidad de Antioquia, Universidad El Bosque, Universidad de Michigan y Universidad de Baylor las palabras utilizadas fueron genéricas y las estrategias de búsqueda las siguientes en inglés y español:

\section{Inglés:}

- Treatment onychophagia.

- onychophagia.

- Nail biting children.

- Onychophagia children.

- Onychophagia intraoral device.

- $\quad$ Bite plate.

- Onychophagia psychology.

- Onychophagia Relaxation.

- Onychophagia competence reaction.
- Onychophagia adverse therapy.

- Nail biting psychology.

- Nail biting relaxation.

- Nail biting competence reaction.

\section{Español:}

- Onicofagia tratamiento.

- Onicofagia terapia.

- Onicofagia rejilla.

- Onicofagia reacción competencia.

- Succión pulgar distracción contingente.

- Hábito succión terapia cognitiva.

- Hábito succión terapia conductual.

- Aparatos Onicofagia.

- Terapia homeopática Onicofagia.

\section{Selección de las investigaciones}

Primero la investigadora, revisó los títulos provenientes de las estrategias de búsqueda y estas fueron introducidas en una tabla creada en Excel.

Posterior a la selección inicial, se realizó lectura de los resúmenes para identificar los artículos relevantes y establecer su calidad. Si no estaba claro si alguna investigación cumplía o no con los criterios de inclusión, entonces se obtuvo el artículo completo para ser analizado, antes de la selección de resúmenes. Una vez que todos los resúmenes potencialmente adecuados para la revisión fueron leídos y seleccionados, se obtuvo el texto completo de los mismos para 
determinar la inclusión, de acuerdo a una tabla estandarizada que consideró tanto la validez interna (científica), como la externa (generalizable).

La investigadora no fue ciega acerca del nombre, autores, instituciones o fuente de publicación, en ningún momento de la revisión.

Inicialmente los estudios fueron juzgados con base en su calidad, de acuerdo a la tabla de Chalmers ${ }^{11}$. La tabla de Chalmers (Tabla 1) está diseñada para ser suficientemente flexible para evaluar la calidad ${ }^{12}$ de ensayos clínicos de cualquier contenido o intervención. Cada ítem en el instrumento de Chalmers tiene un peso de acuerdo a su contribución a la calidad de la investigación que está siendo evaluada.

Los artículos cuyo puntaje según la tabla de Chalmers fue 70 o superior a 70, fueron incluidos en la presente revisión sistemática.

\section{Error del método}

La investigadora fue previamente estandarizada en la lectura de los artículos y en el uso de la tabla de Chalmers con un segundo evaluador experto en lectura crítica.

\section{Análisis de datos}

Los estudios fueron inicialmente categorizados con base en el tratamiento dado a los niños y niñas con onicofagia. Se identificaron cuatro regímenes terapéuticos principales:

- Terapia psicológica: Abordaje terapéutico que busca estimular pensamientos, sentimientos, sensaciones y conocimientos para que el niño (a) deje la práctica del hábito nocivo, en este caso la onicofagia. Estas varían según el trastorno y son la psicoterapia individual, grupal, de autoayuda y rehabilitación neuropsicológica ${ }^{13}$.

- Terapia cognitiva: Es una forma de intervención en la que los patrones de pensamiento adversos, llamados distorsiones cognitivas, son reestructurados por medio de intervenciones psicoeducativas y práctica continua. Se aplica de acuerdo al nivel de conocimiento y capacidad de comunicación con cada individuo $^{14}$

- Aparatos intraorales: Aditamentos instalados para obstruir el posicionamiento de las uñas dentro de la cavidad bucal.

- Terapia conductual: Terapia enfocada a cambiar las conductas y modificar reflejos.

Los datos de los diferentes estudios no fueron comparables, pues los tratamientos usados en los diferentes artículos fueron diferentes. Los tratamientos asignados a los grupos experimentales, no fueron los mismos en las diferentes investigaciones.

Algunos de los estudios analizados, presentaban adecuado objetivo y diseño, pero no había correspondencia adecuada con la metodología utilizada y los resultados.

\section{Datos perdidos}

Se contactó a los diferentes autores para que suministraran los datos faltantes. Cuando los autores no pudieron ser contactados o los datos no fueron suministrados, los datos o los sujetos perdidos, fueron registrados para cada estudio de manera individual. El número de estudios incluido en el análisis final, está reportado en las tabla 2 . 
Tabla 1. Puntajes de Chal

\begin{tabular}{|c|c|}
\hline Categorías & Puntos totales posibles \\
\hline \multicolumn{2}{|l|}{ Evaluación no ciega } \\
\hline Diseño del estudio & 3 \\
\hline Descripción adecuada de la selección de sujetos & 3 \\
\hline Proporción de descripción del tamizaje de pacientes & 3 \\
\hline Descripción de pérdidas y razón de las mismas & 3 \\
\hline Fue definido el régimen terapéutico & 3 \\
\hline Fueron idénticas la presentación del placebo y la droga activa & 3 \\
\hline Fue idéntico el sabor de la droga placebo y la droga activa & 10 \\
\hline Aleatorización ciega & 10 \\
\hline Los pacientes estuvieron ciegos frente al grupo de tratamiento & 4 \\
\hline Los médicos estuvieron ciegos frente al grupo de tratamiento & 3 \\
\hline Los médicos y pacientes fueron ciegos respecto a los resultados & 3 \\
\hline El numero de sujetos necesarios para el ensayo se estimo a priori & 3 \\
\hline Se evaluó la aleatorización en forma adecuada & 3 \\
\hline Se evaluó el ciego en forma adecuada & 3 \\
\hline Fue evaluada la aceptación al tratamiento & 3 \\
\hline Subtotal & 60 \\
\hline \multicolumn{2}{|l|}{ Análisis } \\
\hline Se describen las pruebas estadísticas y valor de $p$ & 3 \\
\hline $\begin{array}{l}\text { Se recalculó el poder estadístico de las diferencias observadas en los ensayos con } \\
\text { resultados negativos }\end{array}$ & 3 \\
\hline Se presenta el intervalo de confianza estimado para el efecto estimado & 2 \\
\hline Se describen tablas de vida o análisis de series temporales & 2 \\
\hline Según indicación se realizó análisis de regresión & 2 \\
\hline El análisis estadístico fue apropiado & 4 \\
\hline Se manejaron apropiadamente las pérdidas & 4 \\
\hline Se describen efectos colaterales y se realizó análisis estadístico para ellos. & 3 \\
\hline Fue apropiado el análisis de subgrupos & 2 \\
\hline El estadístico fue ciego respecto al grupo de tratamiento & 2 \\
\hline Se describen múltiples observaciones de los resultados preliminares & 3 \\
\hline Subtotal & 30 \\
\hline \multicolumn{2}{|l|}{ Presentación } \\
\hline Se describen las fechas de inicio y finalización del consentimiento & 2 \\
\hline Se hizo una análisis inicial para comparar los grupos & 2 \\
\hline Se tabularon todas las mediciones o resultados obtenidos & 4 \\
\hline Presenta curva de supervivencia o proporciona datos suficientes para construirla & 2 \\
\hline Subtotal & 10 \\
\hline Gran total & 100 \\
\hline
\end{tabular}


Tabla 2. Títulos de los estudios seleccionados entre 1985 y 2009, acerca del tratamiento de la onicofagia y que cumplieron los criterios de inclusión

\begin{tabular}{|c|c|c|c|}
\hline Fuente & Autor y año de publicación & $\begin{array}{l}\text { Edad de los } \\
\text { sujetos }\end{array}$ & Diseño \\
\hline $\begin{array}{c}\text { EBSCO, OVID, } \\
\text { MEDLINE, } \\
\text { PUBMED. }\end{array}$ & $\begin{array}{l}\text { Woods DW, Murray LK, Fuqua RW, Seif } \\
\text { TA, Boyer LJ, Siah A: 1999xv }\end{array}$ & $7-9$ & $\begin{array}{l}\text { Ensayo clínico } \\
\text { controlado aleatorizado }\end{array}$ \\
\hline
\end{tabular}

\section{Resultados}

Los resultados del procedimiento para la selección de los artículos, se encuentran en la figura 1.

Se seleccionó el estudio de Woods y col $^{15}$. El puntaje de Chalmers fue 70. El diseño del estudio fue el adecuado, se hizo una adecuada descripción de la selección de los sujetos, el tamizaje de los pacientes fue coherente, el régimen terapéutico fue estrictamente definido y aplicado. No se describen las pérdidas y la razón de las mismas. La aleatorización fue ciega, aunque no fueron ciegos los sujetos, ni los investigadores al tratamiento. El número muestral no fue calculado a priori, no hubo pérdidas durante el seguimiento. El manejo estadístico, la tabulación y las gráficas fueron bien manejados.

En esta investigación, los 26 pacientes incluidos, fueron asignados a uno de los siguientes tres grupos: tratamiento de reversión del hábito con reacción de competencia con conductas similares (cambiar una conducta por otra semejante); tratamiento de reversión del hábito con reacción de competencia con conductas diferentes (cambiar una conducta por otra de diferente tipo) y grupo control. Se tomaron evaluaciones con videos antes y después del tratamiento. Adicionalmente, se evaluó la aceptabilidad social de los pacientes en los grupos de tratamiento. Encontraron, que comparado con el grupo control, los pacientes en terapia disminuyeron el hábito más rápido y de manera más efectiva. Sin embargo, no hubo diferencias significativas al comparar los dos tipos de terapia.

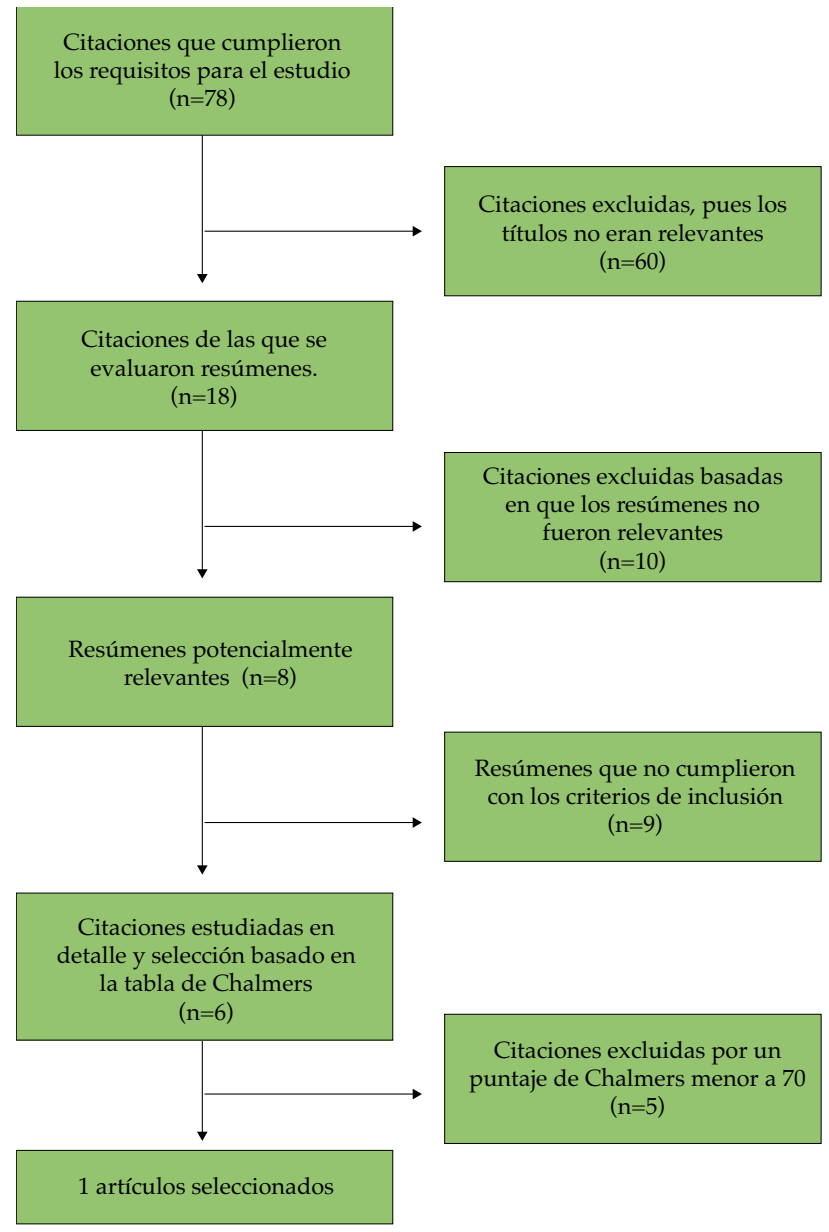

Figura 1. Selección de estudios. 


\section{Discusión}

Todos los seres vivos están expuestos constantemente a múltiples y diversos riesgos de enfermar y de morir. El hombre, que vive en un ambiente sociocultural artificial, es decir, creado y desarrollado históricamente por él mismo, tiene por razones ecológicas y sociales una diversidad grande de riesgos y una oportunidad también mayor de enfrentarse a ellos ${ }^{16}$.

Todos los hábitos tienen su origen dentro del sistema neuromuscular puesto que son patrones reflejos de contracción muscular de naturaleza compleja que se aprenden ${ }^{17}$.

Existen hábitos beneficiosos o funcionales como la masticación, deglución y respiración normal y los incorrectos o deformantes como la respiración bucal, queilofagia, onicofagia, empuje lingual, masticación de objetos y la succión del pulgar $u$ otro dedo (Onicofagia) y uso de chupetes y biberones después de los 3 años, pues antes de esta edad, estos últimos se consideran normales. Estos hábitos deformantes traen como consecuencia alteraciones de la oclusión si se mantienen por largos períodos de tiempo. Otro efecto negativo de estos hábitos pueden ser los trastornos en el lenguaje y en el desarrollo físico y emocional del niño. Este es el motivo por el cual deben ser eliminados tempranamente.

La dieta es primordial para el buen establecimiento de la función. Esta dieta debe ser de consistencia fibrosa para desarrollar adecuadamente el sistema masticatorio. La lactancia materna en los primeros meses de vida favorece el ritmo funcional del complejo bucal, comprendiéndose fácilmente que las ganas de masticar sean despertadas y fomentadas por la alimentación del pecho de la madre, sobre todo si se tiene en cuenta la diferencia entre la alimentación por biberón y por el pecho. Cuando esta lactancia no se da de manera adecuada, pueden aparecer los hábitos parafuncionales bucales ${ }^{18}$.

Se han planteado tratamientos con medicamentos como antidepresivos, cuando hay una enfermedad psiquiátrica asociada, fármacos para la tricotilomanía y el trastorno obsesivo-compulsivo, incluyendo clomipramina ${ }^{19}$, y fluoxetina ${ }^{20}$. Sin embargo aunque estas enfermedades son comunes en adultos, en los niños no se ha probado esta asociación. Debido a esto, las drogas mencionadas tampoco son comúnmente usadas en la población infantil. Es importante señalar que el que la persona esté tomando antidepresivos o antipsicóticos para tratar la onicofagia no quiere decir que el paciente sufra de psicosis.

Otra opción es tomar vitamina B-h (inositol), que reduce las ganas de morderse las uñas por medio del aumento de la serotonina en el cerebro. La serotonina está indicada en el tratamiento de desórdenes compulsivos y similares ${ }^{21}$.

La única terapia para la onicofagia que presenta adecuado sustento en la literatura es la conductual y cognitiva ${ }^{15}$ Dentro de las terapias conductuales, muchos pacientes han encontrado en la terapia de conducta beneficios, tanto por sí solas o como complemento de los fármacos. La primera parte del tratamiento consiste en el cambio de hábitos o terapia de reacción de competencia ${ }^{22}$. Éste es un proceso de cuatro partes que consiste en buscar la forma de eliminar el hábito nocivo y reemplazarlo por otro saludable. Además se aplica una terapia de control de estímulos para identificar y eliminar lo que estimula a cada persona a comerse las uñas.

Los tratamientos para la onicofagia con aparatos, no tienen adecuado soporte en la literatura científica y llama la atención la poca evidencia en general que existe para este tipo de hábito. De hecho, podría afirmarse, que es el hábito oral con menos evidencia disponible para basar la práctica clínica. 


\section{Bibliografía}

1. Tanaka OM, Vitral RW, Tanaka GY, Guerrero AP, Camargo ES. Nailbiting, or onychophagia: a special habit. Am J Orthod Dentofacial Orthop. 2008;134:305-308.

2. Bohne A, Keuthen N, Wilhelm S.Pathologic hairpulling, skin picking, and nail biting.Ann Clin Psychiatry. 2005;17:227232.

3. Ballinger BR. The prevalence of nail-biting in normal and abnormal populations. Br J Psychiatry. 1970;117:445-446.

4. Jabr FI.Severe nail deformity. Nail biting may cause multiple adverse conditions.Postgrad Med. 2005; 118:37-38.

5. Baydaş B, Uslu H, Yavuz I, Ceylan I, Dağsuyu IM. Effect of a chronic nail-biting habit on the oral carriage of Enterobacteriaceae. Oral Microbiol Immunol. 2007;22:1-4.

6. $\quad$ de Berker D. Childhood nail diseases. Dermatol Clin. 2006;24:355-263.

7. Twohig MP, Woods DW, Marcks BA, Teng EJ. Evaluating the efficacy of habit reversal: comparison with a placebo control. J Clin Psychiatry. 2003 Jan;64:40-48.

8. Molina OF, dos Santos J, Mazzetto M, Nelson S, Nowlin T, Mainieri ET. Oral jaw behaviors in TMD and bruxism: a comparison study by severity of bruxism.Cranio. 2001;19:114-122.

9. Lavigne G, Kato T.Usual and unusual orofacial motor activities associated with tooth wear. Int J Prosthodont. 2003;161:80-82

10. Owmann-Moll, P; Kurol, J. European Journal of Orthodontics. 2000; 22: 657- 664.

11. Chalmers TC, Smith H Jr, Balckburn B, Silverman B.. A method for assessing the quality of a randomized control trial. Control Clin Trials 1981;2:31-49.

12. Sonis J, Joines J. The quality of clinical trials published in The Journal of Family Practice, 1974-1991. J Fam Pract. 1994;39:225-235.

13. Bados A, García E, Fusté A. Eficacia y utilidad clínica de la terapia psicológica. Revista internacional de psicología clínica y salud. Int J Clin Health Psychol,2002; 2:477-502.

14. Scott A. Cognitive behavioural therapy and Young people: An introduction. J Fam Health Care. $2009: 19: 80-82$.

15. Woods DW, Murray LK, Fuqua RW, Seif TA, Boyer LJ, Siah A. Comparing the effectiveness of similar and dissimilar competing responses in evaluating the habit reversal treatment for oral-digital habits in children. J Behav Ther Exp Psychiatry. 1999;30:289-300.

16. Slade GO, Caplen DJ. Methodological issuas in longitudinal epidemiologic dental caries. Comunity Dent Oral Epidemiol 1999;27:236-248.

17. Alenen P. Risk in risk definitions. Comunity Dent Oral Epidemiol 1999;27:394.

18. Navarro Nápoles J, Duharte Escalona A. La lactancia materna y su relación con los hábitos bucales incorrectos. MEDISAN. 2003;7 :17-21

19. Leonard HL, Lenane MC, Swedo SE, Rettew DC, Rapoport JL. A double-blind comparison of clomipramine and desipramine treatment of severe onychophagia (nail biting). Arch Gen Psychiatry. 1991;48:821-827.

20. Alexander RC. Fluoxetine treatment of trichotillomania. J Clin Psychiatry. 1991: 34-42

21. Williams, Larsen Kronenberg, Melmed, Polonsky Trastornos del metabolismo del magnesio. En: Tratado de Endocrinología. 10ª Edición, 2003. pp1458-1459. Barcelona.

22. Silber KP, Haynes CE. Treating nailbiting: a comparative analysis of mild aversion and competing response therapies. Behav Res Ther. 1992;30:15-22.

Recibido: 02 -02- 2010

Aceptado: 15 -04- 2010

Correspondencia: martinezresrtrepo@une.net.co 